\title{
Estudio de mercado sobre la utilización de medios digitales como herramientas de información
}

\author{
Market study on the use of digital media as information tools
}

\author{
Jorge Luis González Sánchez \\ Universidad Técnica de Machala \\ jgonzalez@utmachala.edu.ec \\ Machala - Ecuador
}

\author{
Karol Elizabeth Galvez Palomeque \\ Universidad Técnica de Machala \\ kgalvez@utmachala.edu.ec \\ Machala - Ecuador
}

\begin{abstract}
Johan Fabricio Fernández Quinde
Universidad Técnica de Machala

jfernande7@utmachala.edu.ec

Machala - Ecuador
\end{abstract}

\section{Mirka Dayana Córdova Castro \\ Universidad Técnica de Machala \\ mcordova5@utmachala.edu.ec.}

Machala - Ecuador 


\title{
Resumen
}

La presente investigación tiene como objetivo determinar el consumo de medios digitales los tres principales cantones de la provincia del Oro, la metodología que se utilizará para la recolección de datos mediante un método cuantitativo, (encuestas), los resultados según los datos recopilados en los cantones Machala, Pasaje y Santa Rosa se puede observar que el $96 \%$ de la población si utiliza medios digitales para informase, mientras que el $4 \%$ no utiliza. Teniendo como conclusión que la tendencia de consumo de medios digitales en los cantones (Machala, Santa Rosa, Pasaje), en relación al resultado obtenido mediante el instrumento investigativo fue positivo.

\section{Palabras claves}

Redes sociales, información, plataformas virtuales, tecnología.

\begin{abstract}
The present research aims to determine the consumption of digital media in the three main cantons of the province of Oro, The methodology that will be used for data collection through a quantitative method, (surveys), The Results According to the data collected in the Cantons Machala, Pasaje and Santa Rosa it can be observed that $96 \%$ of the population uses digital media to inform themselves, while $4 \%$ does not use it. Taking as a conclusion that the trend of digital media consumption in the cantons (Machala, Santa Rosa, Pasaje), in relation to the result obtained through the research instrument was positive
\end{abstract}

\section{Keywords}

Social networks, information, virtual platforms, technology.

\section{Introducción}

Los medios digitales en la actualidad han alcanzado un nivel alto de importancia en la sociedad, debido a que gracias a ellos lar personas se informan, entretienen y pasan a menudo consumiendo contenido de su interés. (Brock, Carlson, Moilanen, \& Schillo , 2016) 
Los procesos que conllevan a la comunicación se han modificado notoriamente en los últimos años, dando paso a la existencia de una sociedad condicionada y pendiente a la información digital con nuevos comportamientos y formas de interactuar en base a los nuevos escenarios virtuales. (Durante, Martínez, \& Ortiz, 2017)

Según Landeta et al.(2019) los avances drasticos de la tecnologia han cambiado la forma de comunicarse debido que en la actualidad no solo los profesionales en comunicación informan a la ciudadania, sino que el ciudaano comun apoyado con las redes sociales como herramientra fundamental, imparten y comunican diferentes tipos de contenido hacia la sociedad en general.

Por esta razon el siguiente estudio tiene la finanlidad de identificar y comprobar el uso de medio digitales con el fin de informarse, divertirse y otros ambitos comunicativos que se desarrollan normalmente utilizando los medios de comunicación tradicionales como la televisión, la radio, etc. (García, 2016)

La necesidad de una información permanentemente actualizada ha disminuido el tiempo de elaboración de las noticias y, con ello, la investigación y verificación que requiere la información de calidad y sin errores, especialmente cuando proliferan los rumores no contrastados en el espacio público. (Lodoño, Mora, \& Valencia, 2018)

Además, el modelo de negocio de pago por uso, o clickbait, supone que muchas empresas de comunicación tengan pocos incentivos para desempeñar su función de vigilancia y se decanten por la espectacularización. (Quiroga , Murcia, \& Ramírez, 2016)

Este hecho, según los datos disponibles, está deteriorando la confianza de los ciudadanos en la información periodística, por lo tanto, el análisis del objetivo permite determinar los elementos que configuran la credibilidad de la información de actualidad y explorar los argumentos sobre el grado de confianza. (Vidal Férnandez, 2016)

La investigacion se llevó acabo en los tres principales cantones Orences, que son: Machala, Santa Rosa y Pasaje, Tomando en cuenta que actualmente debido a un sinnumero de situaciones fortuitas, que se han desarrrollado en el ambito social, las redes sociales cumplen un papel un rol muy importante como el de impartir contenido, muchas veces exclusivos que no se aprecia en los medios tradicionales, siendo a la vez más comodo para las personas debido a que estos pueden interactuar con dichos medios haciendo que exista un feedback continuo. 
Como medios digitales utilizados en este estudio se escogio a las redes sociales por lo que las personas hoy en día son usuarios de redes sociales las cuales son utilizadas en diferentes dispositivos electronicos y la combinacion de estos dispositivos con las redes sociales logran un mayor alcance al impartir informacion. "En las redes sociales, las personas interactuan entre sí, intercambiando informacion y compartiendo contenidos digitales"' (Marín, 2019).

Como herramienta para el levantamiento de información se ha utilizado encuestas, las cuales han sido aplicadas en los tres cantonnes Orences ya mencionados con anterioridad, tomando en cuenta las diferencia poblacional se aplicó el muestreo correspondiente

\section{Hipótesis}

H1: Los habitantes de los tres principales cantones de la provincia del Oro (Machala, Pasaje y Santa Rosa) tienen un alto consumo de medios digitales para informarse.

\section{Hipótesis Nula}

H0: Los habitantes de los tres principales cantones de la provincia del Oro (Machala, Pasaje y Santa Rosa) no consumen medios digitales para informarse.

\section{Materiales y métodos}

La metodología es uno de los elementos más importantes dentro de la investigación ya este describe cada uno de los métodos aplicados con sus principios, ayuda a conocer la importancia de los mismos y su desarrollo eficiente en los procedimientos. (Castrillón, 2016). Además, que su fin es muy claro, la metodología reside en la descripción de pasos a seguir para llegar a resultados deseados a través de la aplicación de cada uno de ellos. Según Aguilera (2018) explica que el empleo de un método correcto investigativo llevara consigo a cumplir los objetivos establecidos en una investigación.

La metodología que se utilizará para la recolección de datos será mediante un método cuantitativo, se empleará técnicas descriptivas, utilizando la herramienta de cuestionario, misma que será dirigida a una muestra poblacional, conformada por 14 preguntas cerradas, las cuales serán realizadas de forma online mediante google forms y serán enviadas vía correo electrónico, chats, grupos de Facebook y WhatsApp. 
La muestra será calculada mediante un muestreo probabilístico aleatorio de proporción con población conocida con el objeto de tener información relevante que represente el total de consumidores, y sus resultados serán presentados en diagramas de pastel.

Para llevar a cabo el cálculo de la muestra ,se hace uso de la población estudiada en la cual se descartan a personas menores a 15 años ya que no son parte de la muestra, siento un total de 64,431 individuos en el cantón Pasaje, 60,224 en el cantón Santa Rosa, 212,689 en el cantón Machala, teniendo las respectivas muestras de 380 personas encuestadas en el cantón Pasaje,380 en el cantón Santa Rosa y 383 en el cantón Machala, con un nivel de confianza del $95 \%$, del margen de error del $5 \%$, la probabilidad positiva y negativa del 0.5. El tipo de fórmula empleada para el cálculo de la muestra es de carácter cualitativa y de proporción en la cual destacan las siguientes variables:

\section{Formula estadística:}

$\mathrm{N}=$ población 1779

$\mathrm{Z}=95 \%$ de nivel de confianza

$\mathrm{E}=5 \%$ margen de error

$\mathrm{P} \& \mathrm{Q}=$ debido a que no existe un estudio previo la probabilidad negativa (Q) y positiva (P) se mantienen en 0,5 para ambas. (Monzón \& Herrero, 2016

$$
n=\frac{N Z^{2} p q}{(N-1) E^{2}+Z^{2} p q}
$$

\section{Resultados}

y discusión

\section{Género}

\section{a. Masculino $169 \quad 44 \%$ \\ b. Femenino $214 \quad 56 \%$ \begin{tabular}{|l|l|l|} 
TOTALES & $\mathbf{3 8 3}$ & $\mathbf{1 0 0 \%}$
\end{tabular}}

Autor: Los autores

Análisis: En las encuestas realizadas a las 383 personas, arrojó un resultado de que el $56 \%$ fueron personas de género femenino y el $44 \%$ de género masculino.

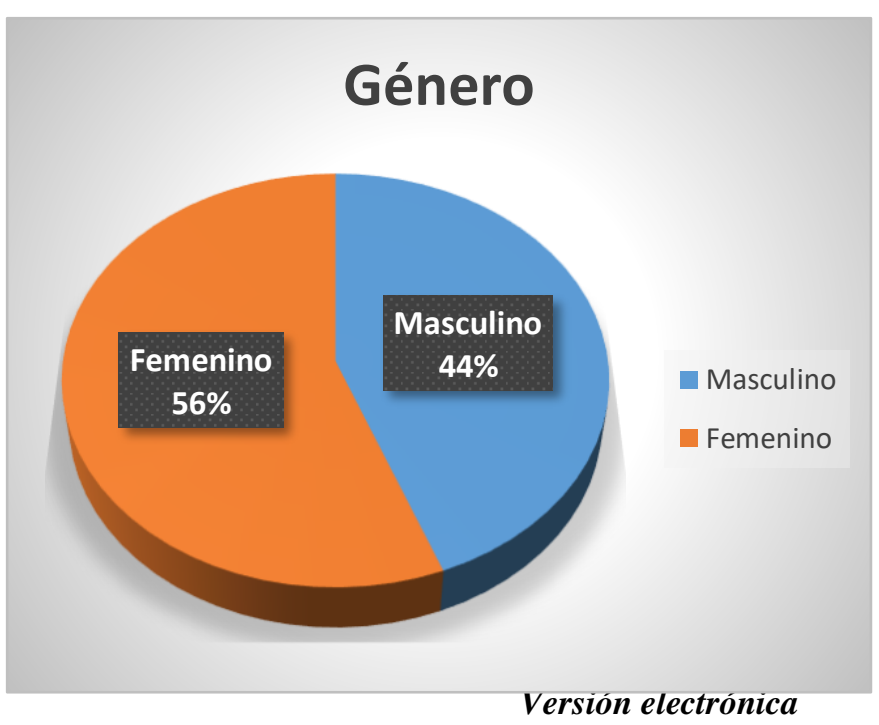

Versión electrónica https://investigacion.utmachala.edu.ec/proceedings/index.php/utmach/issue/view/5 https://doi.org/10.48190/cp.v5n1a14 
Los datos sociodemográficos obtenidos reflejaron que en el cantón Machala el 56\% fueron personas de género femenino y el $44 \%$ de género masculino. Entre los rangos de edad que se pudieron ubicar tenemos que el $37 \%$ de los encuestados tienen entre 26 y 36 años de edad, y el 30\% de 15 a 25 años, en el cantón Pasaje la cual el mayor porcentaje lo poseen las mujeres con el $51 \%$ y complementan el total los hombres con el $49 \%$ y en el cantón Santa Rosa sexo masculino con $61 \%$, siguiendo las mujeres con un 39\%.

\section{Análisis de los resultados}

\section{¿Usted utiliza medios digitales para informarse?}

\section{Tabla}

\begin{tabular}{|c|l|l|}
\hline a. si & 366 & $96 \%$ \\
\hline b. no & $\mathbf{1 7}$ & $\mathbf{4 \%}$ \\
\hline TOTALES & $\mathbf{3 8 3}$ & $\mathbf{1 0 0 \%}$ \\
\hline
\end{tabular}

Autor: Los autores

Análisis: Según los datos recopilados se puede observar que el $96 \%$ de la población si utiliza medios digitales para informase, mientras que el $4 \%$ no utiliza, siendo este el porcentaje menor.

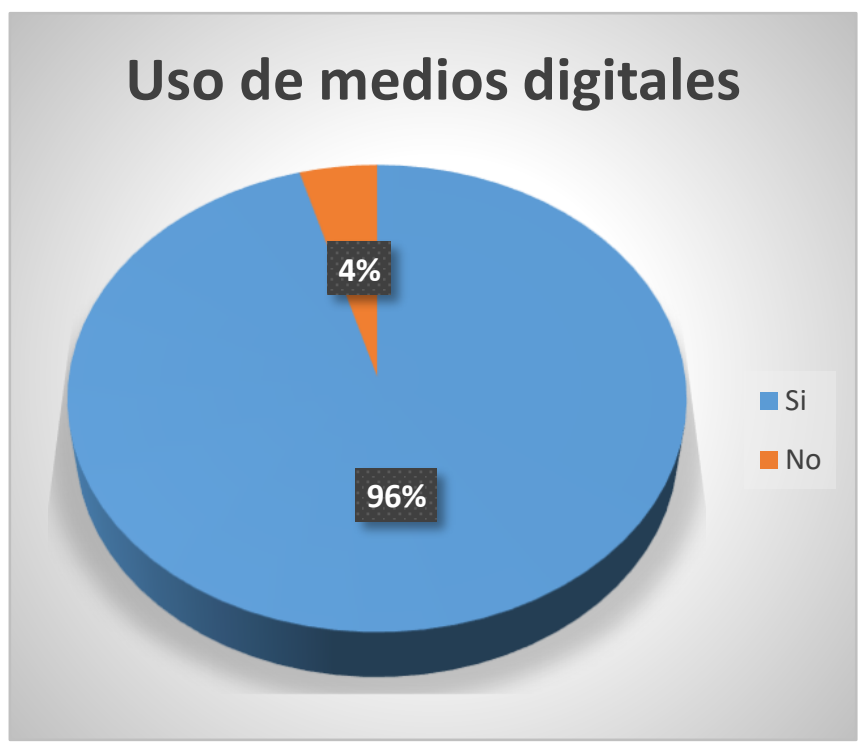

Según los datos recopilados del análisis de uso de medios digitales para informarse en los tres cantones se obtuvo que en el cantón Machala 96\% de la población si utiliza medios digitales para informase, el 99\% de las personas encuestadas en la ciudad de pasaje, y el 94\% en el cantón Santa Rosa.

El uso de medios digitales actualmente es una prioridad en la comunicación y la información, aunque existe una parte de la población que no conoce de ellos, analizando el estudio publicado por (Punín, Martínez, \& Rencoret, 2014) donde se evidencia mediante la aplicación de una metodología de observación y análisis de casos, que los medios de comunicación en plataformas digitales a finales de 2013 y comienzos de 2014 eran pocos, debido a que la mayoría estaba en pleno desarrollo y el usuario generalse encontraba en plena adaptación a la información digital. 
Por ello el consumo de noticas e información online no tenía tanta demanda como la que existe en la actualidad,en donde además cualquier persona con acceso a internet puede convertirse en un comunicador debido al respaldo evidencia, mediante videos o fotografías fáciles de hacer, y de publicación inmediata gracias a la tecnología de los celulares móviles. (Rojo , Bonilla, \& Masaquiza, 2018)

Mientras tanto según estudios realizados por a nivel nacional en el Ecuador determinó que el $70 \%$ de la población hace uso de medios digitales para comunicarse de acontecimientos informativos y para comunicaciones personales. De la misma manera según estudios realizados por más del $45 \%$ de la población mundial hace uso de plataformas digitales como medios de comunicación. Por lo tanto, se considera que los medios digitales han incrementado en gran manera a nivel mundial y la comunicación está inmersa en la internet dejando a un lado algunos de los medios tradicionales informativos.

\section{¿Qué medio digital usted más utiliza para informarse?}

\begin{tabular}{|l|c|c|}
\hline a. Facebook & 382 & $52 \%$ \\
\hline b. YouTube & $\mathbf{4 8}$ & $\mathbf{6 \%}$ \\
\hline c. Instagram & $\mathbf{9 3}$ & $\mathbf{1 3 \%}$ \\
\hline d. twitter & $\mathbf{0}$ & $\mathbf{0 \%}$ \\
\hline e. WhatsApp & $\mathbf{1 1 6}$ & $\mathbf{1 6 \%}$ \\
\hline f. página web & $\mathbf{6}$ & $\mathbf{1 \%}$ \\
\hline g. blogs & $\mathbf{0}$ & $\mathbf{0 \%}$ \\
\hline h. google & $\mathbf{8 7}$ & $\mathbf{1 2 \%}$ \\
\hline $\begin{array}{l}\text { i. } \\
\text { otros............ }\end{array}$ & & \\
\hline TOTAL & $\mathbf{7 3 2}$ & $\mathbf{1 0 0 \%}$ \\
\hline A.
\end{tabular}

Autor: los autores

Análisis: Según los datos obtenidos en las encuestas, entre los medios que más utilizan los ciudadanos para informarse esta Facebook con 52\% y WhatsApp con un valor de $16 \%$, seguido de Instagram con un 13\%, mientras que solo el $1 \%$ utiliza páginas web, siendo este el valor menos de la población encuestada.

\section{Medios que utiliza para informarse}

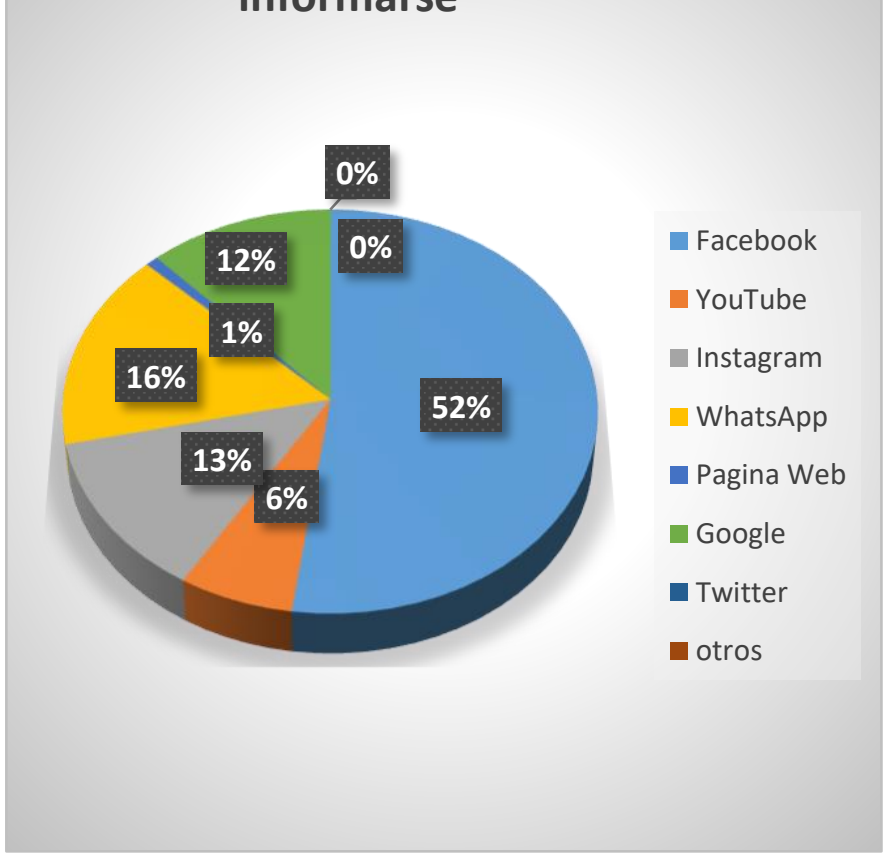


Los ciudadanos del cantón Machala hacen uso de Facebook con 52\% y WhatsApp con un valor de 16\%, mientras tanto el catón Pasaje el 31\% de los usuarios tienen una mayor inclinación por la red social Facebook, luego con el 15\% Instagram y el cantón Santa Rosa el 44\% de la población estudiada usa Facebook como medio digital para informarse mientras que el 30\% usa YouTube.

Facebook mantiene el primer lugar en Ecuador junto a sus marcas Instagram, Messenger y WhatsApp, sumando entre ellas más de 13 millones de usuarios integrados. Instagram mantiene crecimiento por las preferencias de imágenes y videos por parte de los usuarios, y gracias a la variedad de opciones de segmentación que ofrece Facebook. (Schwabe, Fuentes, \& Briede, 2016)

En relación al análisis del entorno social y en comparación a la investigación presentada por (Alvarado \& Herrera, 2020) la cual se enfoca en estudiar el uso de medios digitales en Babahoyo ciudad principal de la provincia de los Ríos, la cual indica que los medios digitales son una herramienta fundamental si de comunicación informativa se trata, puesdebido a su inmediatez las noticias son publicadas y receptadas por los usuarios casi de forma inmediata. Haciendo uso de diferentes plataformas sociales tomando en cuenta que en la pandemia actual aumento su uso, teniendo como resultado de dicha investigación que las redes sociales más utilizadas son Facebook con 65\%, Instagram con un $13 \%$, Whatsapp, $11 \%$, Twitter, y $3 \%$ otras redes sociales. 
¿Razones por las que escoge o seleccione la red social de su preferencia?

\begin{tabular}{|l|l|l|}
\hline Por tendencia & 25 & $7 \%$ \\
\hline Por instantáneo & 13 & $\mathbf{3 \%}$ \\
\hline Por ser confiable & $\mathbf{1 2}$ & $\mathbf{3 \%}$ \\
\hline $\begin{array}{l}\text { Por el material } \\
\text { audiovisual }\end{array}$ & $\mathbf{4 6}$ & $\mathbf{1 2 \%}$ \\
\hline Por facilidad de acceso & $\mathbf{3 4}$ & $\mathbf{9 \%}$ \\
\hline Por el tipo de contenido & $\mathbf{1 5 2}$ & $\mathbf{4 0 \%}$ \\
\hline $\begin{array}{l}\text { Porque permite } \\
\text { interacción }\end{array}$ & $\mathbf{7 4}$ & $\mathbf{1 9 \%}$ \\
\hline Otros & $\mathbf{2 7}$ & $\mathbf{7 \%}$ \\
\hline TOTALES & $\mathbf{3 6 6}$ & $\mathbf{1 0 0 \%}$ \\
\hline
\end{tabular}

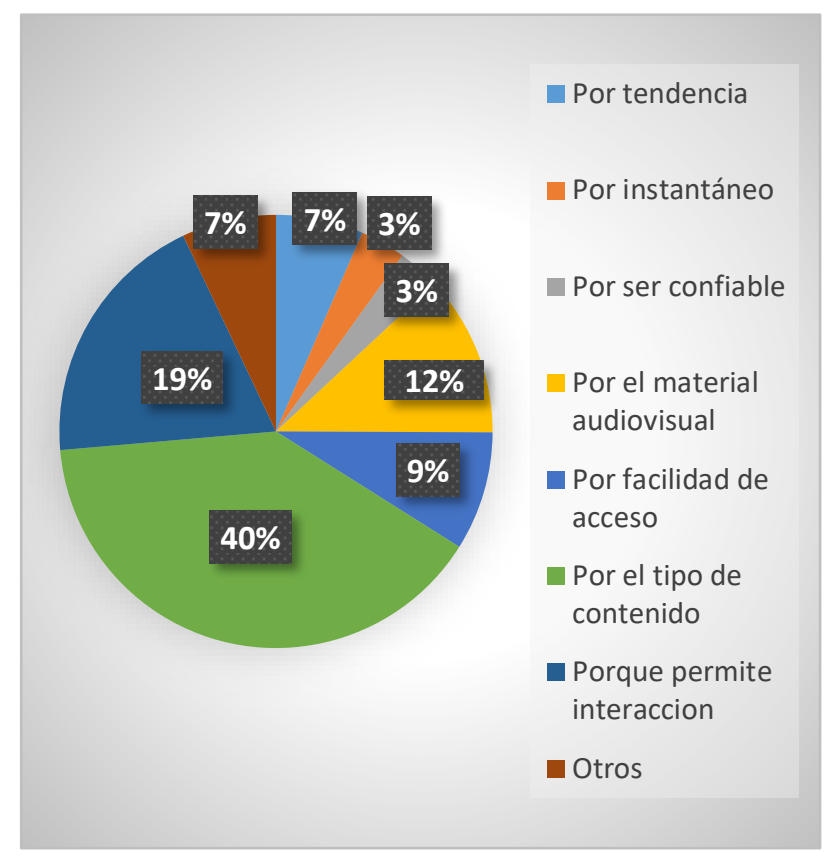

Análisis: Alrededor del $40 \%$ de encuestados seleccionan esta red social por el tipo de contenido que esta posee, el 19\% en cambio por la interacción que permite comunicarse con familiares y amigos y el $12 \%$ por el material audiovisual que los entretiene siendo estos los porcentajes más altos.

Según la encuesta las razones por las que escogen o seleccionan la red social de preferencia en el cantón Machala es alrededor del $40 \%$ por el tipo de contenido que esta posee, el $19 \%$ en cambio por la interacción que permite comunicarse con familiares y amigos, así mismo el cantón Pasaje con el motivo de preferencia más representativo es el de confiabilidad con un $22 \%$ muy a par con el $21 \%$ por contenido y posteriormente en el cantón Santa Rosa se pudo determinar que el $45 \%$ usa los medios digitales de comunicación por su facilidad de acceso, el $29 \%$ por ser confiable.

Según el estudio realizado por (Castillo \& Quezada, 2019). En comparación con la presente investigación. Las personas usan medios digitales con el objetivo de obtener algún tipo de información, muchas veces buscan páginas representadas por empresas de comunicación oficiales. Como son canales de noticias reconocidos. Fijándose siempre en la inmediatez, teniendo este indicador un $50 \%$ en el estudio realizado y los espacios de participación de los usuarios un $25 \%$. Siendo los más representativos en la investigación analizada.

\section{Conclusiones}


- Se pudo determinar que la tendencia de consumo de medios digitales dentro de los tres principales cantones de la provincia de El Oro (Machala, Santa Rosa, Pasaje), en relación al resultado obtenido mediante el instrumento investigativo fue positivo debido a que las personas indicaron a nivel general que utilizan medios digitales para informarse, siendo las redes sociales las principales plataformas para consumir contenido informativo y a su fácil acceso y alcance.

- Por lo tanto, se llega a la conclusión de que los medios de información digitales son de vital importancia para los ciudadanos, ya que brindan noticias actualizadas, al momento con una veracidad relativa que hace que la población esté al tanto de todo lo que pasa en la ciudad y el país; la variedad de noticias también influye bastante e incluso se amplía el segmento al que se quiere llegar. Validando la Hipótesis la cual indica que los habitantes de los tres principales cantones de la Provincia del oro tienen un alto consumo de medios digitales para informarse.

\section{Referencias}

Aguilera, R. (18 de Agosto de 2018). Identidad y diferenciación entre Método y Metodología. Estudios Políticos . (R. Aguilera, Ed.) Estudios Políticos , 9( 28), 81-103. doi:http://www.redalyc.org/pdf/4264/426439549004.pdf

Alvarado, S. R., \& Herrera, F. A. (2020). LOS MEDIOS COMUNICACIÓN DIGITALES Y JÓVENES BABAHOYENSES EN TIEMPOS DE PANDEMIA. MAGAZINES DE LAS CIENCIA, 5, 309-323. Obtenido de https://revistas.utb.edu.ec/index.php/magazine/article/view/1124/812

Brock, B., Carlson, S., Moilanen, M., \& Schillo , B. (Diciembre de 2016). Llegar a los consumidores: cómo la industria tabacalera utiliza el marketing por correo electrónico. ELSEVIER, 4, 103-106. doi:https://doi.org/10.1016/j.pmedr.2016.05.020

Castillo, Y. C., \& Quezada, L. (2019). Entornos digitales y credibilidad de los medios en época de la posverdad. INNOVA Research Journal, 4(2), 90-101. doi:https://doi.org/10.33890/innova.v4.n2.2019.943 
Castrillón, D. (2016). FACTORES CLAVE EN MARKETING ENFOQUE: EMPRESAS DE SERVICIOS. Orbis, 42-58. Obtenido de https://www.redalyc.org/pdf/709/70946593003.pdf

Durante, E., Martínez, J., \& Ortiz, R. (2017). La usabilidad de advergame como estrategia del marketing digital. Quórum Académico, 17(1), 7-10. Obtenido de https://www.redalyc.org/jatsRepo/1990/199062926003/index.html

García, A. (2016). Cultura de servicio en la optimización del servicio al cliente. Telos, 381-398. Obtenido de https://www.redalyc.org/pdf/993/99346931003.pdf

Lodoño, S., Mora, Y., \& Valencia, M. (17 de Agosto de 2018). modelos estadístcos sobre la eficacia del marketing digital. revista EAN(84), 5-19. Obtenido de https://www.redalyc.org/jatsRepo/206/20657075010/index.html

Marín, V. (2019). Las redes sociales en educación: desde la innovación a la investigación educativa. Revista Iberoamericana de Educación a distancia, 22(2), 25 - 33. doi:http://dx.doi.org/10.5944/ried.22.2.24248

Punín, M., Martínez, A., \& Rencoret, N. (2014). Medios digitales en Ecuador:perspectivas de futuro . Comunicar, XXI(42), 199-207. doi: http://dx.doi.org/10.3916/C42-2014-20

Quiroga , D., Murcia, C., \& Ramírez, J. (2016). Internet y su potencial en el marketing estratégico de precios: Una aproximación teórica y. (U. libre, Ed.) Entramado, 12(1), pp. 122-135. Obtenido de https://www.redalyc.org/articulo.oa?id=265447025008

Rojo, M., Bonilla, D., \& Masaquiza, C. (2018). El desarrollo de nuevos productos y su impacto en la producción: caso de estudio BH Consultores. Revista Universidad y Sociedad, 10(1), 134-142. Obtenido de http://scielo.sld.cu/scielo.php?script=sci_arttext\&pid=S221836202018000100134\&lng=es\&tlng=es.

Schwabe, J., Fuentes, P., \& Briede, J. (2016). Caracterización del proceso de diseño de productos de una empresa prestadora deservicios de diseño. Propuesta basada en un enfoque de procesos. Dyna, 83(199), pp. 148-156. Obtenido de https://www.redalyc.org/articulo.oa?id=49648868020

Vargas, B. (2014). TÓPICOS DE INFERENCIA ESTADÍSTICA: EL MÉTODO INDUCTIVO Y EL PROBLEMA DEL TAMAÑO DE LA MUESTRA. (B. Vargas, Ed.) Revista de Difusión cultural y científica de la Universidad La Salle en Bolivia, 7(7), 86-92.

doi:http://www.scielo.org.bo/scielo.php?script=sci_arttext\&pid=S2071081X2014000100007\&lng=es\&nrm=iso

Vidal Férnandez, P. (2016). METODOLOGÍA PARA LA ELABORACIÓN DE UN PLAN DE MARKETING ONLINE. Empresa. Investigación Y Pensamiento Crítico, 5(2), 57-72. doi:https://doi.org/10.17993/3cemp.2016.050226.57-72 
Recibido: 10 Diciembre de 2020 / Aceptado: 16 de agosto de 2021

Conference Proceedings UTMACH V.5 Núm.1 (2021)

ISSN: 2588-056X 